\title{
Experimental investigation on wear resistance of a new cutting fluid using a four ball tribometer - optimization of additive percent
}

\author{
Viorel Paleu*, Viorel Goanţă, and Bogdan Istrate \\ "Gheorghe Asachi" Technical University of Iaşi, Department of Mechanical Engineering, \\ Mechatronics and Robotics, 61-63 D. Mangeron Blvd., Iaşi, Romania
}

\begin{abstract}
The cutting fluid manufacturers aim to obtain the best products at the lowest possible price. To achieve such a compromise experimental work is needed, trying to get the lowest allowed content of additive in the base oil. The paper presents the results of standard tests carried out on four ball machine. This tribometer is used to analyze the load-carrying properties of cutting fluids. The results are compared with the standard requirements, related to the measured wear scar on the three lower balls of the four ball device. An optimum of sulphur additive content into the base oil was found around $10 \%$. According to the actual standards and following the results of carried-out tests, for this specific case the lowest acceptable content of additive is around $4 \%$.
\end{abstract}

\section{Introduction}

A major problem in the metal cutting process is the condition of the cutting tools, influencing the quality of the machined surfaces and the overall machining costs. The dry cutting can generate heat and high temperatures, intensifying the tool wear, increasing the cutting forces and diminishing the surface accuracy of the workpiece.

Three main categories of the cutting fluids are used today: cutting oils, water soluble fluids, and gases. A cutting fluid reduces the friction at tool-chip and tool-workpiece interfaces enhancing the tool life, cools the tool and the chip and facilitates the removal of the chip [1]. To minimize the wear of the tool the cutting fluid have to be a good lubricant, to provide a separating film in the cutting zone.

A good cutting fluid must be able to rapidly eliminate the heat from the tool - workpiece - chip system, especially by convection. For a good flow the cutting fluid is requested to be less viscous, but for high wear resistance it requires to be additived with extreme pressure (EP) additives.

The production cost is an important aspect that interests the cutting fluid manufacturers. It can be minimized by choosing cheap low viscosity base oils and optimizing the quantity of good but expensive EP additives. To achieve such a compromise experimental work is needed, trying to get the lowest allowed content of additive into the base oil.

\footnotetext{
*Corresponding author: vpaleu@yahoo.com
} 
There are different methods to experimentally asses the performances of the cutting fluids. According to ASTM D2783-3, various lubricants performances can be determined on a four ball machine: load - wear index, the weld point, last non-seizure load etc.

A review of the tribological properties of cutting fluids is presented on large in [2]. Some results on four ball machine testing of oil based additived cutting fluids and cutting nanofluids are reported in literature [3-5].

This paper presents the results of standard tests carried out on a four ball machine to analyze the load-carrying properties of cutting fluids. The results are compared with the standard requirements, related to the measured wear scar on the three lower balls of the four ball device.

\section{Materials, testing machine and test conditions}

\subsection{Materials}

The main properties for the base oil, high-sulphur content polysulfide EP additive, and tested balls are indicated in Table 1 .

Table 1. Properties of the base oil, EP additive, and bearing steel balls.

\begin{tabular}{|c|c|c|c|}
\hline Material & Property & Value & Units \\
\hline \multirow{2}{*}{ Base oil } & Kinematic viscosity at $40^{\circ} \mathrm{C}$ & 13.9 & $\mathrm{~mm}^{2} / \mathrm{s}$ \\
\hline & Density at $20^{0 \mathrm{C}}$ & 853 & $\mathrm{Kg} / \mathrm{m}^{3}$ \\
\hline \multirow{5}{*}{ EP additive } & Kinematic viscosity at $25^{\circ} \mathrm{C}$ & 87 & \multirow{3}{*}{$\mathrm{mm}^{2} / \mathrm{s}$} \\
\hline & Kinematic viscosity at $40^{\circ} \mathrm{C}$ & 39 & \\
\hline & Kinematic viscosity at $100^{\circ} \mathrm{C}$ & 6 & \\
\hline & Pour Point & -48 & ${ }^{0} \mathrm{C}$ \\
\hline & Sulfur & 36.9 & $\%$ Weight \\
\hline \multirow{6}{*}{ Steel balls } & Material & \multicolumn{2}{|c|}{ AISI 52100 bearing steel } \\
\hline & Diameter & $1 / 2$ & inch \\
\hline & Density & 7800 & $\mathrm{Kg} / \mathrm{m}^{3}$ \\
\hline & Elasticity modulus & 208 & $\mathrm{GPa}$ \\
\hline & Hardness, HV10 & 700 & $\mathrm{Kg} / \mathrm{mm}^{2}$ \\
\hline & Roughness, $\mathrm{R}_{\mathrm{a}}$ & $0.018 \ldots 0.035$ & $\mu \mathrm{m}$ \\
\hline
\end{tabular}

\subsection{Four ball machine}

A four ball machine is a tribometer designed to test lubricants behaviour in a tribosystem composed by one rotating ball pressed against 3 stationary balls firmly held together and immersed in lubricant (see Figure 1). The four balls touching each other form a pyramid, between the upper and the three lower balls obtaining point contacts.

The four ball machine is composed by an electrical motor and a belt transmission (1), a spindle (2), the four balls testing device (3), the load lamellar spring (4), and a frequency static converter (6). 


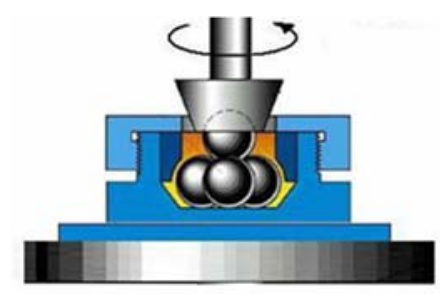

Fig. 1. The testing device of the four ball machine.

Figure 2 presents a general view of the testing machine used in this paper.

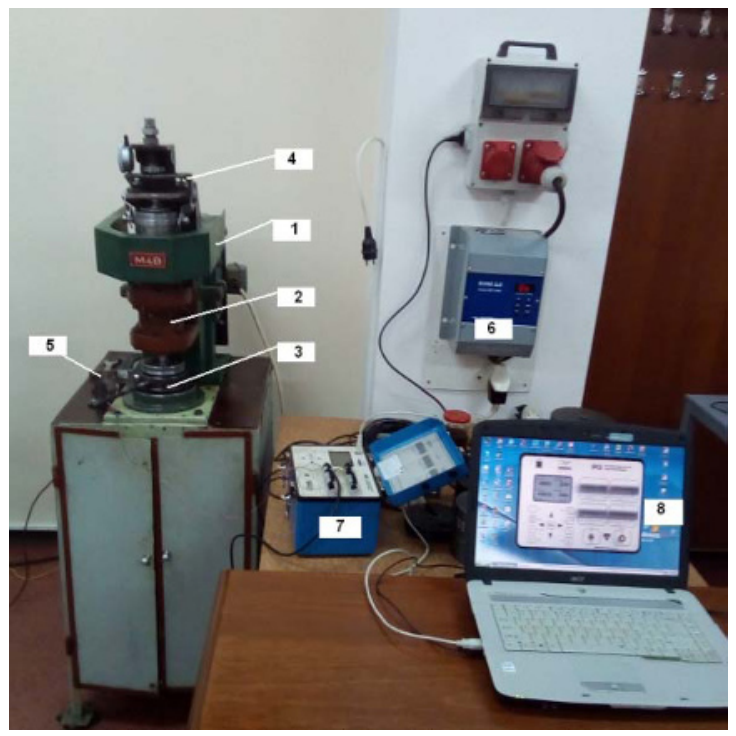

Fig. 2. General view of the four ball machine.

\subsection{Data acquisition system}

The monitored parameter is the friction torque between the upper rotating ball and the three static lower balls. The friction torque created by the friction forces acting on the point contacts between the rotating upper ball and the three stationary balls tries to rotate the testing device. The utilized sensor is resistive, being a metallic elastic leaf (5), with two resistive strain gages brazed on it, attached to the testing device (3). A pin from the frame of the machine blocks the elastic leaf, producing an elastic deformation proportional with the friction torque within the testing device.

The data acquisition system is composed by a P3 - Vishay resistive bridge (7) with four channels, and its software application. The data acquisition system is calibrated by dead weights method. The friction torque values during tests are saved on the internal hard disk of a notebook (8).

The load is maintained constant during tests, by the lamellar spring (4), which was previously calibrated on a compression standard machine.

\subsection{Testing conditions}

The properties of the tested materials are indicated in Table 1. The cutting fluid is obtained by mixing 2 up to 20 percent of EP additive into the base oil, samples of 1 liter being prepared. 
For each test new four unworn steel balls are used, and after their mounting in the testing device about $10 \mathrm{ml}$ of additived oil is added, till the top ball is completely covered. The top ball is clamped to the end of the rotating spindle.

The tests are carried-out according to a specific standard for cutting fluid testing on four ball machines, equivalent to ISO 6743/7 L-MHD and ISO 6743/7 L-MHF, which imposes a mean wear spot measured on the three lower balls of maximum 2 millimeters. The test conditions are: speed of the shaft (rotating upper ball): $1500 \mathrm{rpm}$; constant axial load during tests: $1500 \mathrm{~N}$; time of each test: 1 minute; concentration of the EP additive in the base oil: $2 \% \ldots 20 \%$. In fact, this is a specific test from the general ASTM D2783-3 standard, called compensation scar diameter.

After each test the wear scar diameters on the lower balls were measured and inspected on a microscope at a high magnitude scale.

\section{Results and discussions}

\subsection{Compensation scar diameter}

The compensation scar diameter is defined by ASTM D2783-3 standard as the average diameter, in millimeters, of the wear scar measured after each test on the stationary lower balls. This wear scar is created by the rotating upper ball, pressed against the stationary balls by an axial force, in the presence of a lubricant, without causing scuffing or welding of the balls. Figure 3 presents the compensation scar diameter evolution versus EP additive percent.

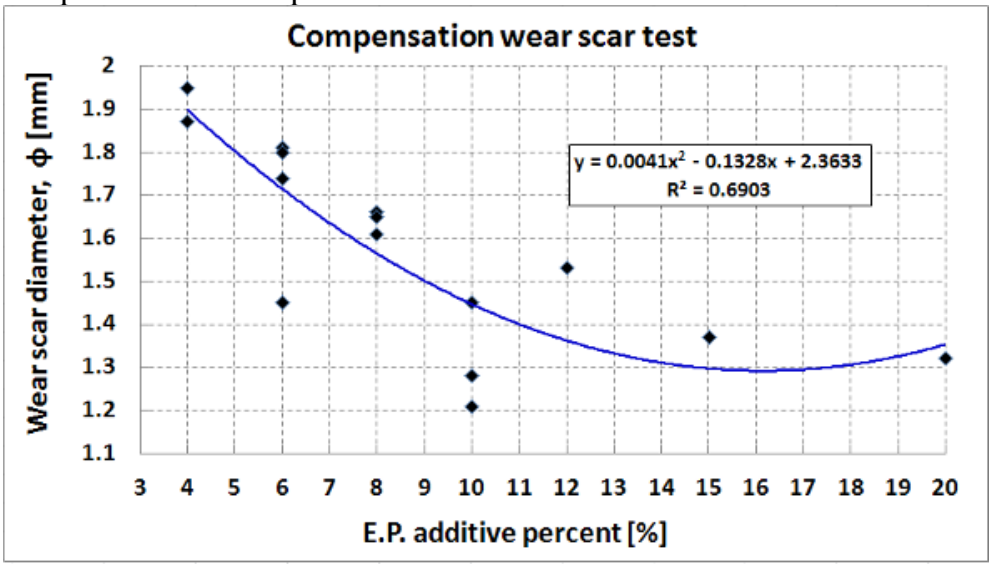

Fig. 3. Mean wear scar diameter versus E.P. additive percent into the base oil.

The wear scar diameter becomes almost constant if the additive percent is increased above 10\%. According to ISO 6743/7 L-MHD and ISO 6743/7 L-MHF standards, the minimum accepted additive percent into the base oil is $4 \%$, the mean wear scar on the three lower balls reaching the limit accepted value of 2 millimeters.

When the obtained results were spread, we repeated up to four times the tests. The variation of the wear scar diameter, $\Phi$, versus EP additive percent, $C$, can be interpolated with a polynomial function of the second order, see equation (1). The $\mathrm{R}^{2}$ parameter is 0.69 , better than for logarithmic $\left(\mathrm{R}^{2}=0.65\right)$, power law $\left(\mathrm{R}^{2}=0.62\right)$, linear $\left(\mathrm{R}^{2}=0.53\right)$, or exponential $\left(\mathrm{R}^{2}=0.52\right)$.

$$
\Phi=0.0041 \cdot C^{2}-0.1328 \cdot C+2.3633
$$




\subsection{Microscopy analysis of the wear scars}

Analyzing the surface of the wear scar by microscopy, it can be observed that no catastrophic wear occurred for the additived samples of oil during the imposed test condition (duration 1 minute, speed $1500 \mathrm{rpm}$, axial load $1500 \mathrm{~N}$ ), for EP additive percent between 4 and 20\%. Figure 4 shows some images for different percent of additive into the base oil.
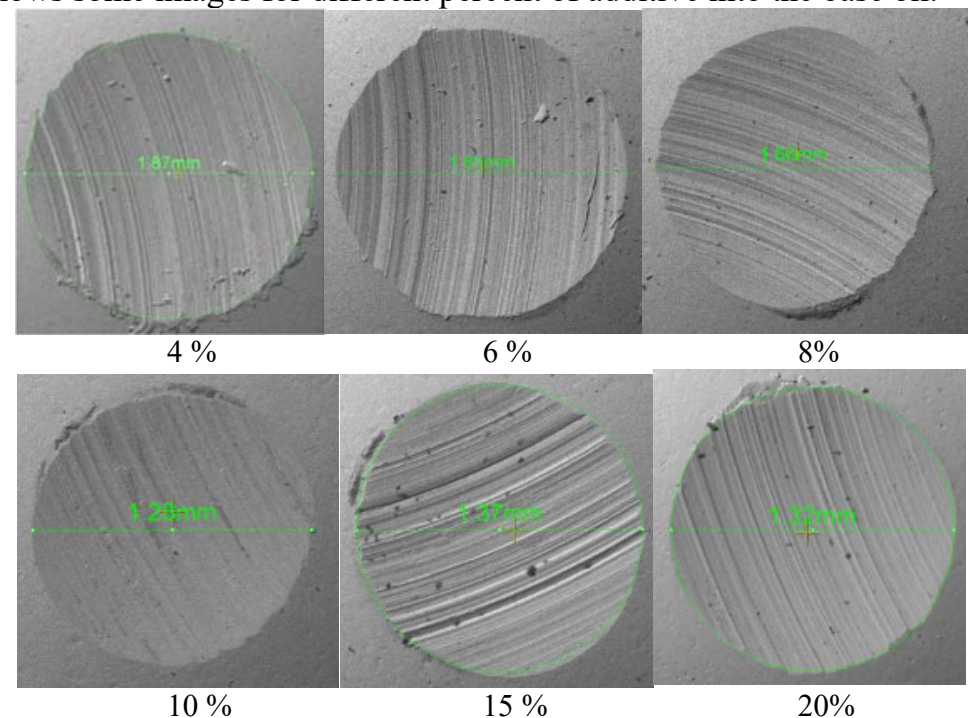

Fig. 4. Images of the wear scars for different percent of additive.

The visual inspection of wear scars shows some wear particles at the border of the scars, and also some lubricant and EP additive scraps.

\subsection{Friction torque evolution}

Friction torque evolution during tests versus EP additive content in the base oil is represented in Figure 5. The friction torque values are distinct at the end of the tests, when the balls lubricated by a sample of oil with $2 \%$ EP additive failed by scuffing. The balls welded together when scuffing appeared (for $2 \%$ of additive), and the friction torque almost doubled its value. The moment when scuffing produced is indicated on Figure 5 by a strong increase of the friction torque. For the rest of the samples the values are very near, being between 2 and $3 \mathrm{~N} \cdot \mathrm{m}$. The monitoring of the four ball machine tests by friction torque evolution is a confident method, allowing the prediction of the occurrence of scuffing in just few seconds.

Analyzing the results, one can be observed that the lowest friction torque and the best surface quality was obtained for $10 \%$ of EP sulphur additive into the base oil. Increasing the content of additive from $10 \%$ to $20 \%$ or decreasing it to $4 \%$, the friction torque augments. But from economical viewpoint the most advantageous case accomplishing the existing standards for cutting fluids wear resistance the optimum is obtained for $4 \%$ of additive. For this percent of additive into the base oil the requirements of the standards are still accomplished, that is the mean wear scar on the lower balls is less than 2 millimeters.

Important savings can be obtained by cutting oil manufacturers by adding just the minimum necessary of additive. 


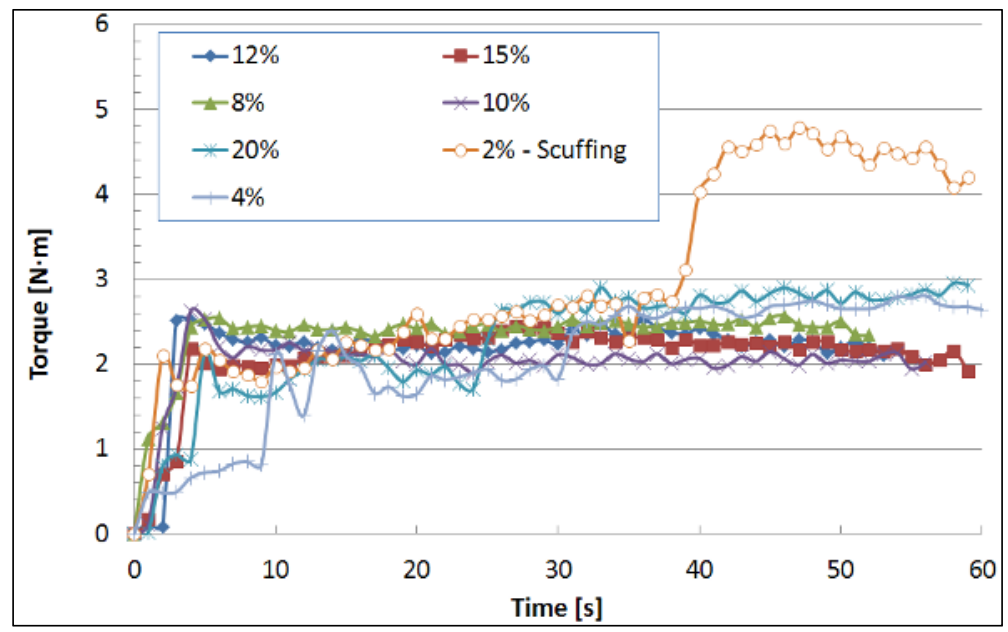

Fig. 5. Friction torque evolution versus EP additive content.

\section{Conclusions}

The tribological properties are very important when developing a new cutting fluid. A good carrying-load of the cutting fluid assures a reduced wear of the tool. The cutting fluid manufacturers must minimize the costs, while accomplishing the requirements of the actual standards. To establish the minimum allowed EP additive concentration into the base oil for new oil based cutting fluid, the compensation wear scar test is developed on four ball tribometer. Analyzing the results, one can be observed that the lowest friction torque and the best surface quality was obtained for $10 \%$ of EP sulphur additive into the base oil, but from economical viewpoint the most advantageous case accomplishing the existing standards for cutting fluids wear resistance the optimum is obtained for $4 \%$ of additive. The monitoring of the four ball machine tests by friction torque evolution is a confident method, allowing the prediction of scuffing occurrence in just few seconds.

Interpolating the obtained experimental results, an empirical formula was found for the dependence between the mean wear scar diameter, measured on the three lower balls, and the EP additive concentration into the base oil. For samples of oil with different percent of additive, the research is intended to be continued with standard incipient seizure load tests.

This work was supported by VIPOIL Company from Iaşi, Romania. The authors also acknowledge Mr. Igor Plugaru, manager of VIPOIL Company, for supplying the technical data on base oil and additives.

\section{References}

1. Y. Isik, Strojniški vestnik, J. Mech. Eng. 563 (2010)

2. A. Anand, K. Vohra, M.I. Ul Haq, A. Raina, M.F. Wani, Tribol. Ind. 384 (2016)

3. A. D. Dongare, A. J. Gite, IOSR J. Eng. 048 (2014)

4. A. D. Dongare, G. J. Vikhe Patil, Int. J. Eng. Resear. Dev. (IJRED) 46 (2012)

5. M. Mosleh, M. Ghaderi, K.A. Shirvani, Jh. Belk, D.J. Grzina, J. Manuf. Proc. 25 (2017) 